\title{
Prediction of IER5 structure and function using a bioinformatics approach
}

\author{
QIANG XIONG ${ }^{1}$, XIAOYAN JIANG ${ }^{1,2}$, XIAODAN LIU $^{3}$, PINGKUN ZHOU $^{3}$ and KUKE DING ${ }^{1,2}$ \\ ${ }^{1}$ National Institute for Radiological Protection; ${ }^{2}$ Key Laboratory of Radiological Protection and Nuclear Emergency, \\ Chinese Center for Disease Control and Prevention, Beijing 100088; ${ }^{3}$ Department of Radiation Toxicology and Oncology, \\ Beijing Institute of Radiation Medicine, Beijing 100850, P.R. China
}

Received October 9, 2018; Accepted March 25, 2019

DOI: $10.3892 / \mathrm{mmr} .2019 .10166$

\begin{abstract}
Immediate-early response gene 5 (IER5) is a gene involved in the regulation of the cell cycle, and its structure and function have been investigated by bioinformatics analyses. The present study determined the sites of promoter methylation and gene ontology (GO) annotations associated with IER5. In addition, we conducted a prediction analysis to determine the physical and chemical properties, hydrophobicity/hydrophilicity, posttranslational modification, subcellular localization, transmembrane structure, signal peptide and secondary and tertiary structures of IER5. One CpG island and several methylated sites were identified close to the promoter of IER5. The GO analysis suggested that IER5 could bind ions and proteins that were mainly associated with metabolic processes. IER5 comprised 327 amino acids and was reported to be an unstable hydrophilic protein with an isoelectric point of 4.91. A total of $18 \mathrm{O}$-glycosylation sites and 22 phosphorylation sites were identified within this protein. The subcellular localization of IER5 was mainly in the nucleus, and its main secondary structural element was the $\alpha$-helix. Bioinformatic analyses of the features of IER5 may improve understanding of its structure and function; however, experimental verification is required.
\end{abstract}

\section{Introduction}

Immediate-early genes encode a type of polypeptides that serve a significant role in cell regulation and the response of the cell to external stimuli. The regulation of the cell cycle by these polypeptides differs among numerous types of cells, due to variations in expression. The response of some immediate-early gene family members to extracellular stimuli is characterized by slow kinetics, which delays transcription

Correspondence to: Professor Kuke Ding, National Institute for Radiological Protection, Chinese Center for Disease Control and Prevention, 2 Xinkang Street, Xicheng, Beijing 100088, P.R. China E-mail: shouding@ccmu.edu.cn

Key words: immediate-early response gene 5, bioinformatics prediction, structure, function and prolongs protein half-life (1). Immediate early response 5 (IER5) was initially reported by Williams et al (1), and is a member of the slow-kinetics immediate-early gene family. IER5 is a gene without introns comprising 2,350 nucleotides and is located in 1q25.3. The predicted open reading frame encodes a 327-amino-acid protein. Its amino terminus is rich in proline residues as previously noted for other homology immediate-early genes, such as pip92/IER2/ETR101. In contrast to pip92/IER2/ETR101, the transcriptional activation of IER 5 does not require induction by phosphokinase C (2).

It has been revealed that IER 5 expression was upregulated following external stimuli, inducing cell apoptosis. Therefore, it may be considered that IER5 is involved in the regulation of the cell cycle. Savitz et al (3), demonstrated that the expression levels of IER5 were increased in peripheral mononuclear cells derived from patients with depression and mood disorders compared with those of healthy subjects. Ishikawa et al $(4,5)$ and Asano et al (6) noted that IER5 was a positive feedback regulator of heat shock factor 1 (HSF1) dephosphorylation, following investigation of the mechanism of HSF1 transcription. In addition, Li et al (7), Kawabata et al (8) and Nakamura et al (9), also observed the cell cycle regulation by IER 5 during the progression of different diseases. Our research group has demonstrated that radiation can induce upregulation of IER5 in tumor cells $(10,11)$ and this process can modulate the transcription of cell division cycle $(C D C) 25 B$ by competitively binding to the $C D C 25 B$ promoter (12). Additionally, we reported that decreased IER5 expression could increase the population of cancer cells in the $\mathrm{G}_{2} / \mathrm{M}$ phase of the cell cycle $(13,14)$, and that binding of a novel transcription factor and GC binding factor (GCF) to the IER5 promoter could act as a negative regulator of IER5 transcriptional activity (15). Furthermore, we proposed that decreased IER5 expression significantly lowered the efficiency of DNA double strand break repair in HeLa cells induced by ionizing radiation (16).

Although various studies have been conducted on the functional mechanism of IER5, only a limited number has explored the structure of the IER5 protein. The present study aimed to determine the structure of the IER5 protein by bioinformatics analysis, and to explain its in vivo function and mechanism of action, based on its structural features, so as to provide a theoretical basis for subsequent experimental determination of its structure. 


\section{Materials and methods}

Sequence of the IER5 gene and protein. The nucleotide sequences of 2,000 bp upstream and 1,000 bp downstream of the transcription sites of the IER 5 gene (Species, Homo sapiens, accession: NC_000001.11, Gene ID: 51278) were downloaded from the National Center for Biotechnology Information (NCBI; https://www.ncbi.nlm.nih.gov/), and the amino acid sequences of the IER5 protein (Entry: Q5VY09, Entry name: IER5_HUMAN, Length: 327-amino-acid) were downloaded from the UniProt database (https://www.uniprot.org/).

Prediction of the IER5 gene sequence. The online software Promoter Scan (https://www-bimas.cit.nih. gov/molbio/proscan/; website decommissioned March 8, 2019) was applied for the prediction of the promoter sequence and the binding sites of the related transcription factors. The program, which recognized $\sim 70 \%$ of primate promoter sequences, predicted promoter regions based on scoring homologies with putative eukaryotic Pol II promoter sequences. The Methprimer (http://www.urogene.org/methprimer/) was applied for the determination of methylation sites and $\mathrm{CpG}$ islands at the promoter region. The criteria for the $\mathrm{CpG}$ island prediction results were the following: Island size $>100, \mathrm{GC} \%$ (percentage of $\mathrm{G}$ plus $\mathrm{C})>50 \%$ and observed/expected values $>0.5$. Gene Ontology (GO) and annotations were investigated using the GO Enrichment Analysis using the AmiGO tool (http://amigo. geneontology.org/amigo/landing). GO enrichment analysis identified relevant groups of genes that functioned collectively, which reduced the thousands of molecular changes to notably fewer biological functions in order to describe a putative function corresponding to the mean number of molecular changes.

Prediction of IER5 protein features. The physical and chemical properties of the IER5 protein were predicted by the ProtParam tool (https://web.expasy.org/protparam/). The protein parameters, including the molecular weight, theoretical isoelectric point, amino acid composition, atomic composition, extinction coefficient and instability index were calculated based on either compositional data or on the $\mathrm{N}$-terminal amino acid residues. The hydrophobicity/hydrophilicity of the IER5 protein was predicted by the ProtScale (https://web.expasy.org/protscale/) which provided 57 scales defined by a numerical value assigned to each type of amino acid. The most frequently used scales were the hydrophobicity or hydrophilicity scales and the secondary structure conformational parameters scales. The O-glycosylation sites were predicted by a genetic engineering approach. The NetOGlyc 4.0 Server (http://www.cbs. dtu.dk/services/NetOGlyc/) was used enable a proteome-wide discovery approach of O-glycan sites by a 'bottom-up' ETD-based mass spectrometric analysis. The $\mathrm{N}$-glycosylation sites of the IER5 protein were predicted by the NetNGlyc 1.0 Server (http://www.cbs.dtu.dk/services/NetNGlyc/) which was based on an artificial neural network in an attempt to discriminate between glycosylated and non-glycosylated sequences. The phosphorylation sites of the IER5 protein were predicted by the NetPhos 3.1 Server (http://www.cbs. dtu.dk/services/NetPhos/) which predicted serine, threonine and tyrosine phosphorylation sites in eukaryotic proteins using ensembles of neural networks and 17 kinases as follows:
Ataxia telangiectasia-mutated, casein kinase (CK)I, CKII, calmodulin-dependent protein kinase-II, DNA-dependent protein kinase catalytic subunit, epidermal growth factor receptor, glycogen synthase kinase (GSK)3, insulin receptor (INSR), protein kinase A (PKA), protein kinase B, protein kinase C (PKC), cGMP-dependent protein kinase, ribosomal S6 kinase, SRC, cyclin-dependent kinase 1 (cdc2), cyclin-dependent kinase 5 (cdk5) and p38 mitogen-activated kinase (MAPK). The subcellular localization of the IER5 protein was predicted by the PSORT II tool (https://psort.hgc. $\mathrm{jp} /$ form $2 . \mathrm{html}$ ) from its amino acid sequences. The location of the transmembrane, intracellular and extracellular regions was predicted by the TMHMM Server v2.0 (http://www.cbs.dtu. $\mathrm{dk} /$ services/TMHMM/) by reading a FASTA-format protein sequence. The presence and location of signal peptide cleavage sites in the amino acid sequences were predicted by the SignalP 4.1 Server (http://www.cbs.dtu.dk/services/SignalP/) with a D-cutoff score of 0.5 . The nuclear localization sequence (NLS) of the IER5 protein was predicted by the NLS mapper (http://nls-mapper.iab.keio.ac.jp/cgi-bin/NLS_Mapper_form. cgi) with a cut-off score of 4.0. The secondary and tertiary structures of the IER5 protein were predicted by the PSIPRED (http://bioinf.cs.ucl.ac.uk/psipred/) and I-TASSER (https://zhanglab.ccmb.med.umich.edu/I-TASSER/) software, respectively. These two methods used a known amino acid sequence to match a template in a protein database. All-by-all TM scores were calculated for the full set of putative templates and the matrix of scores was analyzed to remove any possible outlying templates whose structure was too dissimilar with that of the full set of templates. The tertiary structures of the IER5 protein was compiled and produced by PyMOL (https://pymol.org).

\section{Results}

Promoter binding and methylation analysis of the IER5 gene. The sequences located at 2,000 bp upstream and 1,000 bp downstream of the transcription sites of IER 5 were analyzed, and the promoter was located at a region between 1,722 and 1,972 bp in the plus-strand (Table I). We identified one $\mathrm{CpG}$ island located at a region between 1,499 and 2,944 bp and several potential methylation sites (Fig. 1). In addition, the predicted promoter sequence of IER 5 overlapped with the $\mathrm{CpG}$ island. We further examined the transcription factors sites close to the promoter region and identified specific transcription factors sites associated with methylation, such as the AP-2 (Table II).

Physical and chemical properties and hydrophobicity/hydrophilicity of the IER5 protein. A total of $~ 12.84 \%$ (42/327) of the amino acids (Asp and Glu) were identified with negative charge, whereas $9.48 \%$ (31/327) of the amino acids (Arg and Lys) were identified with positive charge. The IER5 protein contained 11 Cys residues. Considering that all Cys residues formed cystines, the estimated molar extinction coefficient in the aqueous solution was $32,595 \mathrm{M}^{-1} \mathrm{~cm}^{-1}$, whereas for $0.1 \%$ absorbance $(1 \mathrm{~g} / 1)$ would be 0.967 ; however, providing all Cys residues could not form cystines, the corresponding value would be 31,970 and 0.949 . The IER 5 protein was predicted as an unstable protein, of which the structural formula was $\mathrm{C}_{1459} \mathrm{H}_{2294} \mathrm{~N}_{428} \mathrm{O}_{464} \mathrm{~S}_{14}$ and the total molecular weight was 
Table I. Promoter prediction of immediate early response 5 gene.

\begin{tabular}{|c|c|c|c|c|}
\hline Promoter & Start & End & Score & Sequence \\
\hline \multirow[t]{6}{*}{ Promoter 1} & 1,722 & 1,972 & 72.54 & AATCTGTAACTCCAAACAGTAGCGCTCTCGAGCACCGTCCCGA \\
\hline & & & & ACATTCACTCCCCACGGGCCTGGTCTGCGGCCGCAAGCCGTC \\
\hline & & & & GCCCCCTTTAAGAGCCTGCTCCGCGGGACTAACGTTCGAACG \\
\hline & & & & GGCCCTGGCGCCCCTCCTCGGGCTCCGATTGGCCGTGCGCGG \\
\hline & & & & CGCACGAGGGCGCGCCGGCCAGCCCCGGAACGGTTGGCGGC \\
\hline & & & & CCTTCGTGATTGGCGGTCGAGAAGCCTATATAAGGCGCGGG \\
\hline
\end{tabular}

Table II. Transcription factor sites about the promoter of immediate early response 5 .

\begin{tabular}{lccc}
\hline Name & Strand & Location & Weight \\
\hline AP-2 & + & 1773 & 1.355 \\
AP-2 & + & 1774 & 1.108 \\
UCE.2 & + & 1793 & 1.278 \\
UCE.2 & - & 1796 & 1.216 \\
GCF & + & 1856 & 2.361 \\
CTF & + & 1875 & 1.704 \\
UCE.2 & + & 1879 & 1.278 \\
NFI & - & 1881 & 4.221 \\
junB-US2 & - & 1882 & 1.51 \\
TTR_inverted_repeat & - & 1892 & 3.442 \\
GCF & - & 1893 & 2.284 \\
UCE.2 & - & 1910 & 1.216 \\
AP-2 & - & 1930 & 1.672 \\
HNF1 & - & 1931 & 1.012 \\
TFIID & + & 1958 & 1.971 \\
GCF & + & 1968 & 2.361 \\
T-Ag & + & 1970 & 1.086 \\
\hline
\end{tabular}

AP-2, activating protein 2; CTF, CCAAT box-binding transcription factor; GCF, GC-rich sequence DNA-binding factor; HNF1, homeobox A; junB-US2, JunB proto-oncogene, AP-1 transcription factor subunit; NFI, nuclear factor I; TFIID, transcription factor II $\mathrm{D}$; UCE, ubiquitin-conjugating enzyme; $\mathrm{T}-\mathrm{Ag}$, large tumor antigen.

estimated to 33703.69. The theoretical isoelectric point was 4.91, of which the coefficient of instability was 61.1.

The aliphatic index of the IER 5 protein was estimated to 60.4 and the average hydrophilic value was estimated to -0.493 . Leu, the most hydrophobic amino acid, was identified at the 38th position and with an index value of 1.944 . The most hydrophilic amino acid was reported at the 293th position and its index was -2.976 . According to the hydrophilic/hydrophobic distribution diagram (Fig. 2), the majority of the amino acids were hydrophilic amino acids, and therefore IER5 was considered a hydrophilic protein.

Posttranslational modification of the IER5 protein. Protein modifications, such as glycosylation and phosphorylation are required to fulfill protein physiological function.

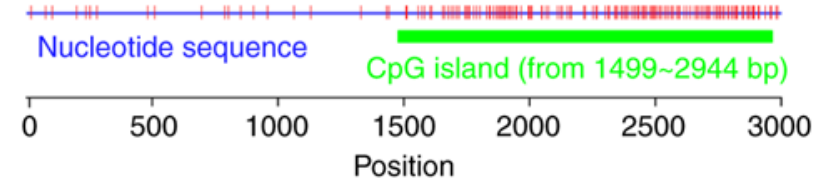

Figure 1. Methylation and $\mathrm{CpG}$ island prediction of immediate early response 5. The blue line denotes the nucleotide sequence, while the short vertical red lines indicate $\mathrm{CpG}$ sites; and green cross coarse line denotes the $\mathrm{CpG}$ island within the indicated region.

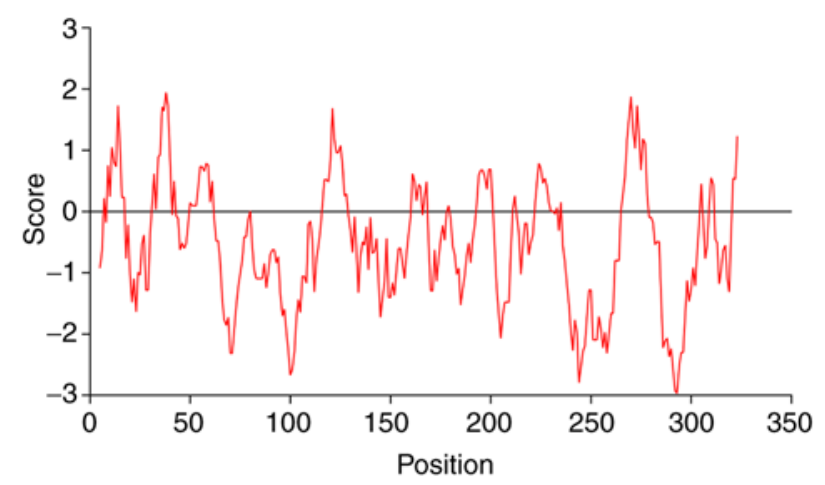

Figure 2. Hydrophobicity/hydrophilicity of the IER5 protein. Positive scores presented hydrophobicity and negative scores indicated hydrophilicity. The higher the absolute value, the higher the degree of hydrophobicity/hydrophilicity.

Glycosylation serves an important role in the interaction between proteins and other macromolecules (17). A total of $18 \mathrm{O}$-glycosylation sites were identified (score $>0.5$ ), in the IER 5 protein; however, no N-glycosylation sites were reported. Phosphorylation is required for signal transduction (18). The results indicated 15 serine (Ser), six threonine (Thr) and one tyrosine (Tyr) protein kinase phosphorylation sites (score $>0.5$, Fig. 3). A total of 9 kinases associated with the phosphorylation reactions, including PKA, cdc2, INSR, PKC, cdk5, GSK3, p38MAPK, CKII and CKI were reported in addition to 'unspecified' types.

Subcellular localization, transmembrane structure and signal peptide identification of the IER5 protein. The prediction of the subcellular localization of IER5 suggested that the protein exhibited a $56.5 \%$ probability of localizing in the nucleus, whereas the possibility for cytoskeletal and mitochondrial localization was notably lower (17.4 and $13.0 \%$, respectively). The prediction indicated a lower potential for localization in 


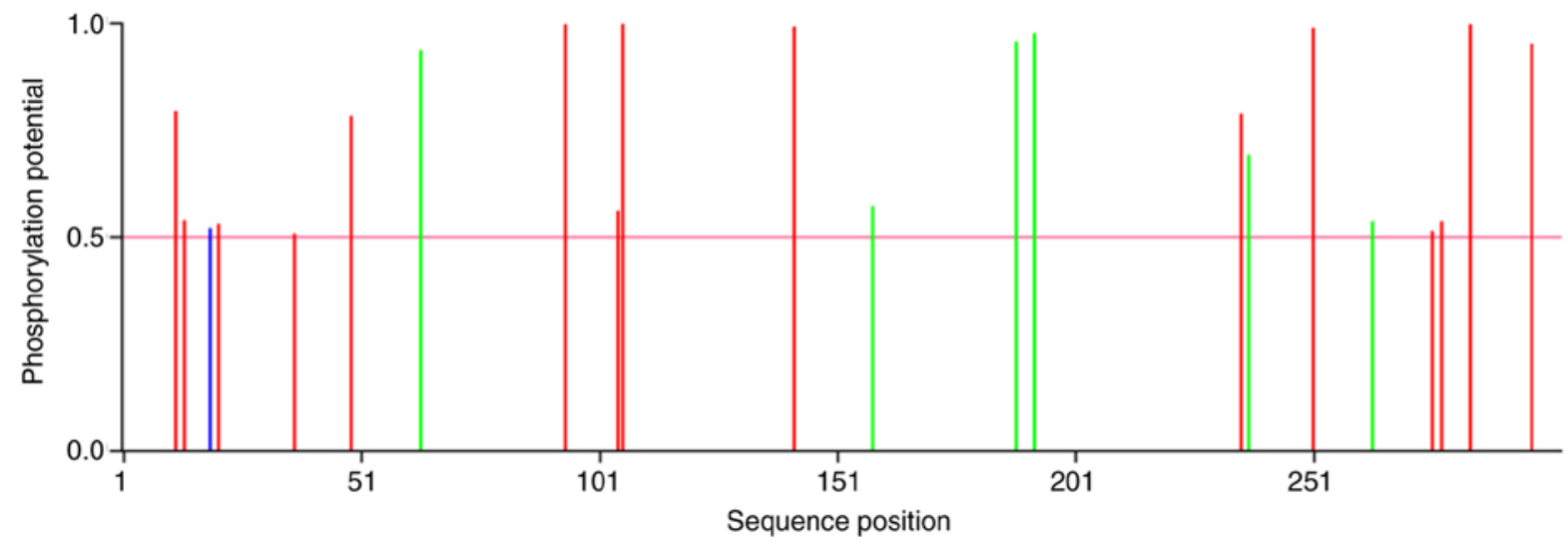

Figure 3. Phosphorylation site of the immediate early response 5 protein. Red lines denote serine phosphorylation sites, green lines indicates threonine phosphorylation sites, the blue line denotes a tyrosine phosphorylation site and the pink line indicates the threshold.

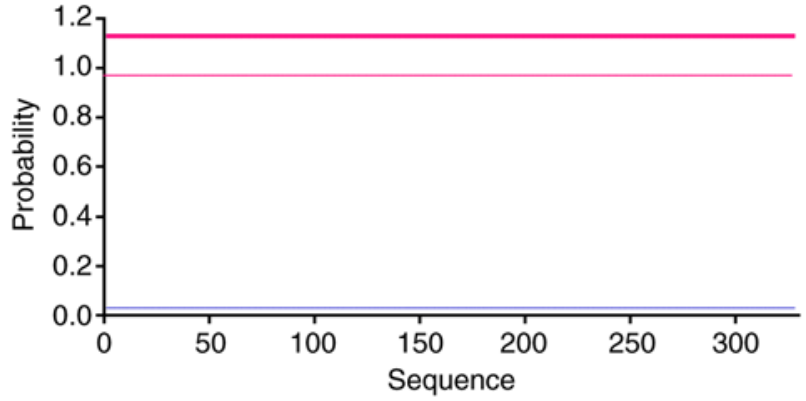

Figure 4. Transmembrane structures of the immediate early response 5 protein. The dark purple line denotes the IER5 protein, the lower purple line indicates the outer cell membrane and the blue line represents the inner cell membrane.

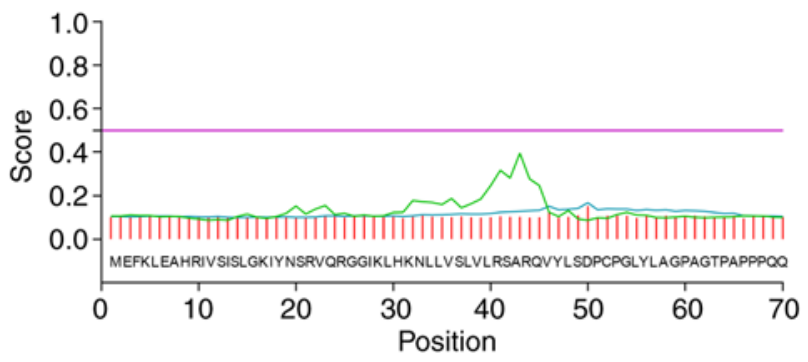

Figure 5. Signal peptides of the immediate early response 5 protein. The red lines denote the $\mathrm{C}$-score, green line indicates the $\mathrm{S}$-score and the blue line denotes the Y-score.

the mitochondria, Golgi apparatus and vesicular secretion system (4.3\%). No transmembrane structures (Fig. 4) and signal peptides (Fig. 5) were present. Further analysis indicated that the IER5 protein may possess a nuclear localization sequence (NLS) GSTPLKKPRRNLE (the position in protein sequence from the $\mathrm{N}$ terminal to $\mathrm{C}$ terminal is $235-247$ ) with a score of 4.5 (threshold of 5.0).

Secondary structure of the IER5 protein. The secondary structure refers to a periodic structure arranged along a direction, and is the regular repeated conformation in the protein polypeptide chain. PSIPRED has been previously used to predict protein secondary structure based on a two-stage

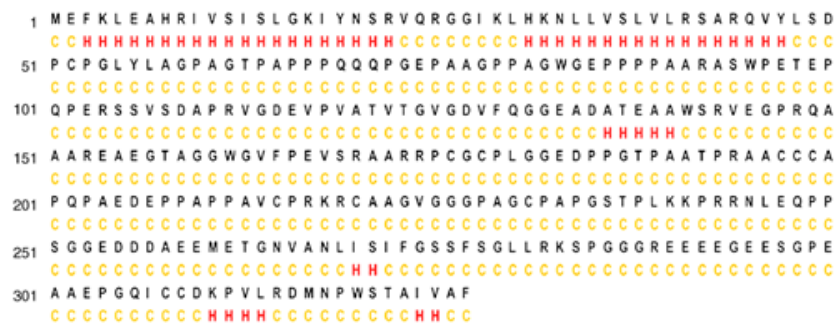

Figure 6 . Secondary structure of the immediate early response 5 protein. $\mathrm{H}$ denotes $\alpha$-helix and $\mathrm{C}$ denotes disordered coil states.

neural network; the average prediction accuracy was estimated at a range of $76.5-78.3 \%$ (19). The results revealed 6 $\alpha$-helixes, but no $\beta$-sheet or $\beta$-turn motifs. The remaining structural parts of the proteins were determined to present as disordered coils (Fig. 6).

Tertiary structure of the IER 5 protein. I-TASSER is an online integrated platform based on the 'sequence-structure-function' model of automatic protein structure and function prediction. Starting from the amino acid sequence, the platform produces the three-dimensional atomic-scale model through the comparison of multiple threading alignment approaches and the iterative structural assembly simulation $(20,21)$. The platform presents five models following the completion of predicting the tertiary structure; default model 1 is considered as the best model based on comprehensive analysis of the three parameters, namely the C-score, the TM score and the RMSD. The tertiary structure was presented in a cartoon model embedded in the surface mode (Fig. 7).

GO of the IER5 gene. GO is a database established by the GO consortium, which is a unified induction, interpretation and analysis of the cytological components, molecular functions and biological approaches of genes and their products. We searched for GO terms and annotations associated with IER5 by the AmiGO browser. The present study reported that this gene was involved in several primary biological and metabolic processes that require ion and protein binding. The main distribution of IER 5 has not been predicted in terms of cellular components (Table III). 


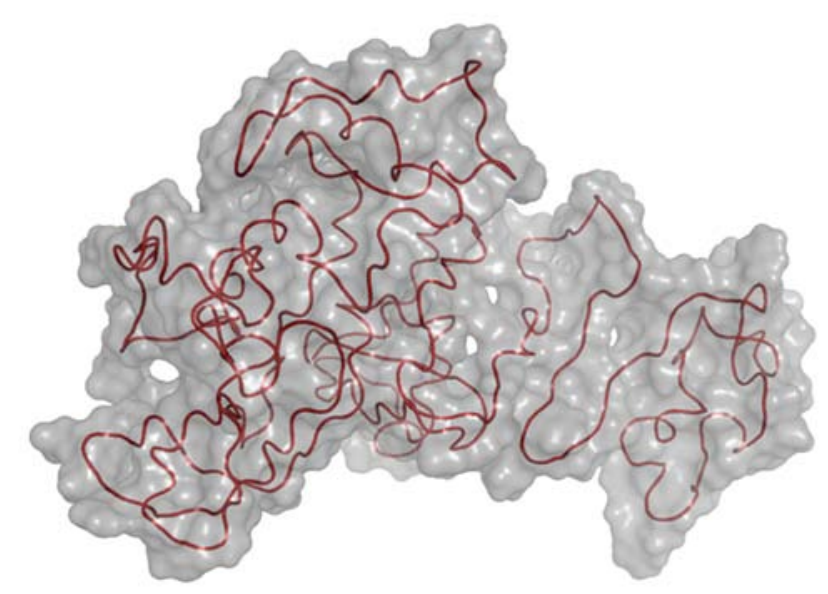

Figure 7. Tertiary structure of the immediate early response 5 protein.

Table III. GO of immediate early response 5.

\begin{tabular}{|c|c|c|}
\hline Ontology & GO ID & Term \\
\hline Biological process & GO:0044238 & $\begin{array}{l}\text { Primary metabolic } \\
\text { process }\end{array}$ \\
\hline Cellular component & - & \\
\hline \multirow[t]{3}{*}{ Molecular function } & GO:0043167 & Ion binding \\
\hline & GO:0042802 & $\begin{array}{l}\text { Identical protein } \\
\text { binding }\end{array}$ \\
\hline & GO:0005515 & Protein binding \\
\hline
\end{tabular}

-, not predicted. GO, Gene Ontology.

\section{Discussion}

In the present study, we investigated the structure and function of IER5, and its encoded protein using bioinformatics online analysis software. Hypermethylation of $\mathrm{CpG}$ islands at the promoter region has been reported to inhibit the transcriptional activity of the gene, whereas low promoter methylation activates gene expression $(22,23)$. The results of the present study indicated one $\mathrm{CpG}$ island and several potential methylation sites. Liu et al (10) and Shi et al (11) demonstrated that radiation could upregulate the expression levels of IER5. Therefore, we proposed that the methylation levels of the wild type IER5 gene may be low, but could notably increase following radiation exposure, inducing its expression $(24,25)$. Specific transcription factors have been located at the promoter region of IER 5 and certain protein binding sites were suggested by GO analysis. Our previous study reported two GCF binding sites at the promoter region, which is in agreement with the present findings (15). Following the binding of GCF, the transcriptional activity and radiation sensitivity of IER 5 significantly decreased (15).

Glycosylation serves an important role in cellular immunity, signal transduction, protein translation regulation and protein degradation. For example, the majority of transcription factors and enzymes require glycosylation following translation (17). In addition, phosphorylation serves a key role in protein signal transduction, gene expression and cell cycle regulation (18). The present study reported $18 \mathrm{O}$-glycosylation sites and 22 phosphorylation sites in the IER5 protein, which reflected the complexity of IER5 protein function.

Based on the prediction of protein subcellular localization, transmembrane region and signal peptide identification, it was speculated that the IER5 protein was mainly localized in the nucleus. The absence of the transmembrane structure and of the signal peptide indicated that the IER5 protein did not require entry into other membrane organelles. Following protein expression, various hydrophilic structures and lack of the transmembrane structure and of the signal peptide may facilitate the free diffusion of the IER5 protein in the cell without its modification by the endoplasmic reticulum or the Golgi apparatus $(26,27)$. The IER5 protein may be channeled from the nuclear pore complex to the nucleus possibly via an NLS.

The helix-turn-helix domain (HTH) is a relatively conserved structure with various patterns that correspond to different protein families (28). HTH contains two $\alpha$-helixes, which are connected by one turn, and can recognize the specific base sequence of the DNA in order to regulate its transcription, replication and translation (29). Previous studies suggested that radiation increased the expression levels of the IER5 gene and protein $(10,11)$. Competitive binding of IER5 to the $C d c 25 B$ promoter led to downregulated expression levels of Cdc25B (12). We demonstrated that the secondary structure of IER5 had only $6 \alpha$-helixes. Following structure prediction, it was speculated that IER5 could possess an HTH structure based on its function. Further experiments are required to confirm this hypothesis.

Of note, the present study has certain limitations as only bioinformatics predictions were conducted. Furthermore, we did not conduct investigations using clinical samples, which may verify the results reported in the present study.

We examined the features of the IER5 gene and protein using bioinformatics analyses, which could aid future investigation of their biological functions. Furthermore, predicting the IER5 may provide a experimental basis for investigation into its functions in the future.

\section{Acknowledgements}

Not applicable.

\section{Funding}

The present work was supported by grants from the National Natural Science Foundation of China (grant nos. 31170806, 31770907 and 31640022) and the Beijing Natural Science Foundation (grant no. 7172146).

\section{Availability of data and materials}

All data generated or analyzed during the present study are included in this published article.

\section{Authors' contributions}

QX, XJ, XL, PZ and KD conceived and designed the study. QX wrote the paper. All authors reviewed and edited the manuscript. 


\section{Ethics approval and consent to participate}

Not applicable.

\section{Patient consent for publication}

Not applicable.

\section{Competing interests}

The authors declare that they have no competing interests.

\section{References}

1. Williams M, Lyu MS, Yang YL, Lin EP, Dunbrack R, Birren B, Cunningham $\mathbf{J}$ and Hunter $\mathrm{K}$ : Ier5, a novel member of the slow-kinetics immediate-early genes. Genomics 55: 327-334, 1999.

2. Takaya T, Kasatani K, Noguchi S and Nikawa J: Functional analyses of immediate early gene ETR101 expressed in yeast. Biosci Biotechnol Biochem 73: 1653-1660, 2009.

3. Savitz J, Frank MB, Victor T, Bebak M, Marino JH, Bellgowan PS, McKinney BA, Bodurka J, Kent Teague T and Drevets WC: Inflammation and neurological disease-related genes are differentially expressed in depressed patients with mood disorders and correlate with morphometric and functional imaging abnormalities. Brain Behav Immun 31: 161-171, 2013.

4. Ishikawa Y and Sakurai H: Heat-induced expression of the immediate-early gene IER 5 and its involvement in the proliferation of heat-shocked cells. FEBS J 282: 332-340, 2015.

5. Ishikawa Y, Kawabata S and Sakurai H: HSF1 transcriptional activity is modulated by IER5 and PP2A/B55. FEBS Lett 589: 1150-1155, 2015.

6. Asano Y, Kawase T, Okabe A, Tsutsumi S, Ichikawa H, Tatebe S, Kitabayashi I, Tashiro F, Namiki H, Kondo T, et al: IER5 generates a novel hypo-phosphorylated active form of HSF1 and contributes to tumorigenesis. Sci Rep 6: 19174, 2016.

7. Li XN, Ji C, Zhou PK and Wu YM: Establishments of IER5 silence and overexpression cervical cancer $\mathrm{SiHa}$ cell lines and analysis of radiosensitivity. Int J Clin Exp Pathol 9: 6671-6682, 2016.

8. Kawabata S, Ishita Y, Ishikawa $Y$ and Sakurai $\mathrm{H}$ : Immediate-early response 5 (IER5) interacts with protein phosphatase $2 \mathrm{~A}$ and regulates the phosphorylation of ribosomal protein S6 kinase and heat shock factor 1. FEBS Lett 589: 3679-3685, 2015.

9. Nakamura S, Nagata Y, Tan L, Takemura T, Shibata K, Fujie M, Fujisawa S, Tanaka Y, Toda M, Makita R, et al: Transcriptional repression of $\mathrm{Cdc} 25 \mathrm{~B}$ by IER 5 inhibits the proliferation of leukemic progenitor cells through NF-YB and p300 in acute myeloid leukemia. PLoS One 6: e28011, 2011.

10. Liu Y, Tian M, Zhao H, He Y, Li F, Li X, Yu X, Ding K, Zhou P and $\mathrm{Wu}$ Y: IER5 as a promising predictive marker promotes irradiation-induced apoptosis in cervical cancer tissues from patients undergoing chemoradiotherapy. Oncotarget 8: 36438-36448, 2017.

11. Shi HM, Ding KK, Zhou PK, Guo DM, Chen D, Li YS, Zhao CL, Zhao CC and Zhang X: Radiation-induced expression of IER5 is dose-dependent and not associated with the clinical outcomes of radiotherapy in cervical cancer. Oncol Lett 11: 1309-1314, 2016.

12. Yang C, Yang M, Feng Z, Liu X, Yin L, Zhou P and Ding K: Radiation modulated the interaction of IER5 protein and CDC25B promoter DNA in primary hepatocellular carcinoma. Int J Clin Exp Pathol 9: 2888-2895, 2016.
13. Yang C, Wang Y, Hao C, Yuan Z, Liu X, Yang F, Jiang H, Jiang $\mathrm{X}$, Zhou $\mathrm{P}$ and Ding K. IER5 promotes irradiation- and cisplatin-induced apoptosis in human hepatocellular carcinoma cells. Am J Transl Res 8: 1789-1798, 2016.

14. Ding KK, Shang ZF, Hao C, Xu QZ, Shen JJ, Yang CJ, Xie YH, Qiao C, Wang Y, Xu LL and Zhou PK: Induced expression of the IER 5 gene by gamma-ray irradiation and its involvement in cell cycle checkpoint control and survival. Radiat Environ Biophys 48: 205-213, 2009.

15. Yang C, Yin L, Zhou P, Liu X, Yang M, Yang F, Jiang H and Ding K: Transcriptional regulation of IER5 in response to radiation in HepG2. Cancer Gene Ther 23: 61-65, 2016.

16. Yu XP, Wu YM, Liu Y, Tian M, Wang JD, Ding KK, Ma T and Zhou PK: IER5 is involved in DNA double-strand breaks repair in association with PAPR1 in Hela cells. Int J Med Sci 14: 1292-1300, 2017.

17. Ohtsubo K and Marth JD: Glycosylation in Cellular mechanisms of health and disease. Cell 126: 855-867, 2006

18. Singh V, Ram M, Kumar R, Prasad R, Roy BK and Singh KK: Phosphorylation: Implications in cancer. Protein J 36: 1-6, 2017.

19. Jones DT: Protein secondary structure prediction based on position-specific scoring matrices. J Mol Biol 292: 195-202, 1999.

20. Yang J and Zhang Y: I-TASSER server: New development for protein structure and function predictions. Nucleic Acids Res 43: W174-W181, 2015.

21. Zhang Y: I-TASSER: Fully automated protein structure prediction in CASP8. Proteins 77 (Suppl 9): S100-S113, 2009.

22. Hashimshony T, Zhang J, Keshet I, Bustin M and Cedar H: The role of DNA methylation in setting up chromatin structure during development. Nat Genet 34: 187-192, 2003.

23. Balada E, Ordi-Ros J, Serrano-Acedo S, Martinez-Lostao L, Rosa-Leyva $\mathrm{M}$ and Vilardell-Tarrés $\mathrm{M}$ : Transcript levels of DNA methyltransferases DNMT1, DNMT3A and DNMT3B in CD4+ T cells from patients with systemic lupus erythematosus. Immunology 124: 339-347, 2008.

24. Illingworth RS and Bird AP: CpG islands-'a rough guide'. FEBS Lett 583: 1713-1720, 2009.

25. Eckhardt F, Lewin J, Cortese R, Rakyan VK, Attwood J, Burger M, Burton J, Cox TV, Davies R, Down TA, et al: DNA methylation profiling of human chromosomes 6,20 and 22. Nat Genet 38: 1378-1385, 2006.

26. Palmer KJ, Konkel JE and Stephens DJ: PCTAIRE protein kinases interact directly with the COPII complex and modulate secretory cargo transport. J Cell Sci 118: 3839-3847, 2005.

27. Bard F, Mazelin L, Péchoux-Longin C, Malhotra V and Jurdic P: Src regulates golgi structure and KDEL receptor-dependent retrograde transport to the endoplasmic reticulum. J Biol Chem 278: 46601-46606, 2003.

28. Wintjens R and Rooman M: Structural classification of HTH DNA-binding domains and protein-DNA interaction modes. J Mol Biol 262: 294-313, 1996.

29. Aravind L and Koonin EV: DNA-binding proteins and evolution of transcription regulation in the archaea. Nucleic Acids Res 27: 4658-4670, 1999 .

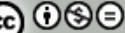

This work is licensed under a Creative Commons Attribution-NonCommercial-NoDerivatives 4.0 International (CC BY-NC-ND 4.0) License. 